\title{
Learning Mechanics Principles by Playing Sport
}

\author{
${ }^{1}$ Díez-Jiménez E., 1Martínez-Muñoz M., ${ }^{2}$ Muñoz Jiménez, J., ${ }^{1}$ Valiente-Blanco I., ${ }^{3}$ Gómez- \\ García M.J. \\ ${ }^{1}$ Universidad de Alcalá, ${ }^{2}$ Universidad de Extremadura, ${ }^{3}$ Universidad Carlos III de Madrid
}

\begin{abstract}
Most of high-school students have typically lower assimilation of the basic concepts of mechanics in comparison with other subjects which are closer to their daily life. In addition, the technical language fairly used in mechanics lessons is far away from their common vocabulary, thus generating estrangement and lack of interest. On the other hand, high-school students are in general attracted and familiarized with sports and sports terminology. The idea hereby proposed is trying to get high-school students closer to mechanics by integrating concepts of mechanics and technical language in their sport activities. In this work, an innovative experimental and demonstrative pedagogical methodology is described. The methodology is based on two main pillars: embracing concepts while playing and normalizing technical language during pleasant moments. The work provides several activities descriptions and procedures as well as suggestions of technical language, uses to mechanics and sports high-school teachers in order to achieve a better inclusion of mechanics in high-school students.
\end{abstract}

\section{Introduction}

Practical and experimental work is essential in studies in science. It contributes to deeply learn and assimilate scientific contents, which are mostly based on evidences and experiments (try-error). This is key in any teaching-learning process [1]. However, most of the time devoted to mechanics occurs inside the classroom following theoretical explanations or solving exercises. Although, laboratory lessons are a good complement, the availability of labs and the final total time spent in the labs is reduced. In addition, the required technical language and the mathematical complexity presents mechanics as a difficult and not useable in high-school student daily life matter [2].

Thus, the reality of the majority of mechanics high-school classrooms is that they learn new concepts, passes their exams but those concepts remain as theoretical knowledge kept in the drawer. This fact leads to a disregard on mechanics. In last years, multiple teachers and education systems directors have noticed a worrying lack of interest and assimilation on mechanics contents during highschool education period [3], [4].

However, mechanics is really in a lot of common daily life events. It is important to identify those events and application cases in order to encourage students to understand their daily phenomena by knowing mechanics [5]. Even more, the future of professional careers of most of students will be highly linked to technological or scientific new developments, even if the students choose a social science, politics or arts. Therefore, familiarizing technical language from mechanics, or other scientific subject, can be very convenient to prevent the negative feeling towards their future compulsory approach to these fields [6], [7]. Thus, it is recommendable to work during high-school period in order to revert the disinterest on mechanics [8], [9].

Therefore, new pedagogical methodologies proposed must take into account those statements. Two main work targets can be defined: improve the familiarization to technical language and improve the assimilation of physical concepts. Following these targets, the contents of the mechanics subject could be adapted or include the new methods in future physic educational programs.

\section{Approach: Mechanics through physical education}

As in other related sciences, the active learning of mechanics has been a widely debated issue for decades prior to the university level. More specifically, in Europe, this aspect has been mainly due to the impulse of methodological renewal promoted by the Bologna Process [10]. One important debate forum of students and teachers takes place each summer at CERN during its Summer Students Programme. There are many students and professors who can carry out stays and research in the laboratories of CERN, from the baccalaureate stage to doctoral programs. Both teachers and students who have participated in these activities have the same opinion; mechanics is a subject whose curriculum has not changed in many years and although new methodologies are developed, the situation related to 
the interest and assimilation of concepts does not improve.

Focusing on Spain, as a close-to-authors example, the teaching of scientific disciplines in general, and of mechanics in particular, is experiencing in recent years a decrease in the number of weekly hours devoted, [11]. Moreover, a decrease in effort and predisposition on the part of students has been noticed. The subject is taught one year earlier but partially since mechanics is shared with chemistry. Even worse is the fact that mechanics is an optative subject from 15-16 years old generates a decrease on the level and skills acquired for the students each course.

The mechanics contents are very dense and cumbersome, it does not leave time to develop and deepen into the basics; it is focused on improper concepts of the level at which it is directed and it does not focus on what the student can do and understand. It is common that a student, a few days after of a test, cannot keep a minimal conversation about what he learned or not even repeat the same test. Faced with this situation, teachers must use creative methods to make it more understandable, but they are limited to actions in the classroom and for a very limited time.

Besides from the pitfalls cited in the previous paragraph, another problem is the agenda of the current Spanish Law of Education, LOMCE [11], which is based on classical mechanics. It is only during the last course before university, when the index of contents includes waves, electromagnetism, optics, relativity, nuclear and quantum. Many of the students discover in this course what mechanics really is. They find difficult to understand how main general physical rules can be applied to can be applied to particular cases.

On the contrary, sports in general and the subject of physical education in particular is one of the most attractive, motivating and fun subject for students. It can be taught mainly outside the classroom and students can acquire the main competencies of the curriculum regardless of their major or minor sports skills. Many studies in physical education have sought to identify and categorize the modes of student interaction in order to gain greater insight into the nature of cooperative activity. More others recent studies have examined how modes of interaction evolve on the basis of the modes of collective activity that they generate. These studies have shown to describe and explain the interactions among individuals and the processes they generate, which then lead to the construction, deconstruction or reconstruction of different interaction modes [12]. These methods can be directly applied to mechanics learning through sports.

In addition, the rate of sports practice during the time of secondary education is very high and popular. Sport is usually presenting in the lives of almost all students in some way or another. Whether in extracurricular activities, in sports clubs or simply during their leisure time, sport is a central theme. Likewise, sports language is understood, used and even created by adolescents. Mechanics teachers can take advantage of this attraction and use sports as a vehicle to introduce concepts of mechanics and technical language into the lives of our students.

\section{Goals \& Layout of this work}

The main objective of the methodology herby presented is to improve the learning of students in the subject of Mechanics through the practice of sports and by using a proper language of sport sciences. In this way, students will be able to know the application of Mechanics in everyday life, mainly in sports, increasing the development of physical skills, and promoting the knowledge and understanding of the science involved in a physical activity and human movement.

The work can be used as a teacher's book for applying the proposed methodology. It is divided in two parts: proposals of physical education exercises linking with concepts of mechanics and proposal of translation from sport language and terms to technical language.

In the first part, we present a complete didactic unit. We describe in detail exercises to be carried out by the physical education teacher, the mechanics teacher or jointly. These are simple exercises, with games and in which we relate the actions with the explanation of the physical phenomena that take place.

In the second part, we present a list of technical vocabulary equivalent to slang words or sports language. The purpose of this compendium is that the teachers, and therefore the students, change their sports and colloquial language by the precise technical language. If this learning is done during a period of play or sports practice, in the medium term it will be more familiar to the student and thus less boring.

\section{Embracing concepts of physics while playing}

Each country has a series of laws through which the syllabus of high school is established. The regulation of the elements that determine the teaching and learning processes for each of the subjects is also provided: objectives of each educational stage, competences, contents, didactic methodology, evaluable learning standards, results and evaluation criteria.

A common factor in all the syllabus is that mechanics plays a central role in the intellectual development of students. Mechanics shares with the rest of the disciplines the responsibility of promoting 
the acquisition of the necessary skills to be to integrated into the society actively.

As a scientific discipline, it has the added commitment of providing students with specific tools allowing to face the future with guarantees, participating in the economic and social development to which the scientific, technological and innovative capacity of society itself is linked. In order for these expectations to materialize, the teaching of this subject should encourage a contextualized learning that relates the concepts with the historical evolution of scientific knowledge; that establishes the relationship between science, technology and society; that enhances verbal argumentation; the ability to establish quantitative and spatial relationships, as well as the ability to solve problems with precision and rigor.

In order to carry out the teaching and learning process of mechanics a methodological system especially relevant in this matter is proposed below. This methodology consists of learning mechanics from physical education and sports within the high school periods.

Every teaching-learning process must start from a rigorous planning of what is intended to be achieved, having clear which are the objectives or goals, what resources are necessary, which didactic methods are the most appropriate and how the learning and feedback evaluated is.

We present the concepts, competences and evaluable standards for an effective teaching of mechanics in high school students which will be developed by means of sports activities. More specifically, we present a teacher's guide for teaching the next contents:

- Speed - uniform linear motion

- Acceleration - uniform accelerated motion

- Rotation - uniform circular motion

- Law of inertia: newton's first law

- Fundamental law of dynamics: Newton's second law

- Law of action and reaction: Newton's third law

- Friction force and coefficient of friction

- Impulse

- Fluids: air - aerodynamic drag and Magnus force

It is recommended to respect the order of the contents since one topic may be needed for the subsequent ones.

Speed - Uniform linear motion: The concept of speed of a body moving uniformly can be very well understood through running exercises.

Competencies: Differentiate the magnitudes necessary to describe the movement: position, time and speed. Distinguish clearly between position, time and speed units. Understanding the concept of relative motion.

Facilities / Materials: athletics track or sports court, chronometer, flexometer

\section{Exercises / Plays:}

1. Measure and mark a distance around 10-20 meter. Start a race between two students while other two students measure the time employed in running the distance. Running students must initiate the run several meters before to start the measuring time with a constant speed. Each pair of students must calculate the average speed in the 10-20 meters track. They have to compare which has larger average speed. Both in $\mathrm{m} / \mathrm{s}$ and $\mathrm{km} / \mathrm{h}$ values must be calculated and compared with typical car speeds. Variants: longer tracks, competitions in teams.

2. Relative motion. One student must grab the flexometer from its start and the other from its end (figure 1). Both students must run together, trying to keep the flexometer at a fixed initial distance. This distance must be registered at the beginning and at the end of the exercise. If there is a difference, it means that there has been a relative motion between students. The value of the difference will be used to calculate their relative speed.

Variants: competitions between couples.

Learning results: To determine correctly an average speed in different units. To calculate relative speeds.

Assessment: Correct calculations of average speeds in $\mathrm{m} / \mathrm{s}$ and in $\mathrm{km} / \mathrm{h}$.

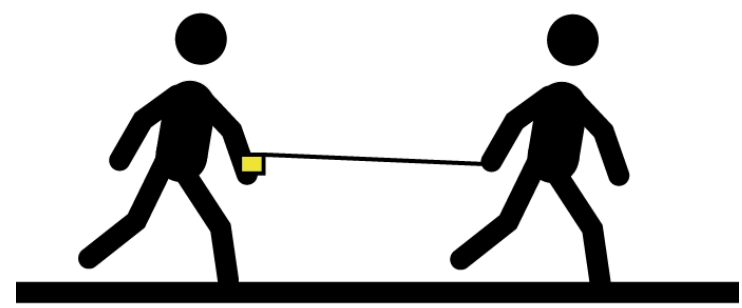

Figure 1. Students running with a flexometer to demonstrate relative motions.

Acceleration - uniform accelerated motion: The concept of acceleration of a body may be easily understood by using a skateboard and a playground slope while having fun.

Competencies: Differentiate the magnitudes necessary to describe an accelerated movement: 
position and time. Distinguish clearly between position, time and acceleration units. To understand the concept of relative motion.

Facilities / Materials: playground slope, chronometer, flexometer

\section{Exercises / Plays:}

1. Measure and mark a distance around 10-20 meter on the slope. One of the students sits down on the skateboard at the top of the slope (figure 2). A second student triggers the motion and measures the total time of the motion. The experiment can be repeated by switching the students. Once the time is correctly determined, the constant acceleration value can be determined from uniform accelerated motion equation. The results can be compared with the gravitational acceleration for analysis. Variants: different slopes will give different accelerations, repeat the exercise but with shorter or longer tracks lengths to realize that acceleration is independent of the displacement.

Learning results: To determine correctly an acceleration in different slopes.

Assessment: Correct calculations of accelerations $\left(\mathrm{m} / \mathrm{s}^{2}\right)$.

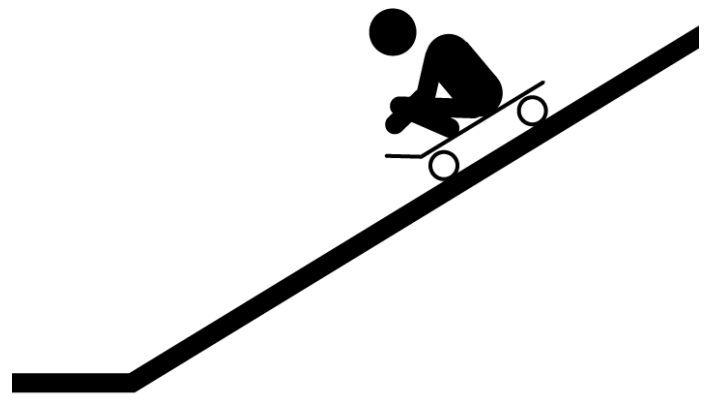

Figure 2. Student falling on the skate.

Rotation - uniform circular motion: Rotational motion is easy to understand for students but what is harder is to relate rotation motion with linear motion. Next exercises are proposed to link both concepts.

Competencies: Relate linear motion with rotational motion. Distinguish clearly between position, time and circular motion units. Understand the concept of circular motion
Facilities / Materials: athletics track or sports court, chronometer, rope

\section{Exercises / Plays:}

1. Locate one student in the center of the circle and a second student in the radius, 3 4 meters is enough. Both students must hold the rope. The student at the radius initiates a jogging while pulling the rope. In this way the student will initiate a circular motion. Once the circular motion is constant, a third student will measure the time gone by for several rotations. With these measurements, the circular speed can be calculated. Variants: Different rope lengths.

2. Again, one student acts as pivot and a second student runs in the radius. Once the constant rotation is set, suddenly the student in the center release the rope; thus, the second student starts to run linearly with the same speed. A third student measures the time and distance from the release and calculates the linear speed. Then, they relate the rotational motion with linear motion using the radius as a factor, and noticing the relationship.

Variants: shorter rope, faster speeds.

Learning results: To determine correctly a circular speed in different units (rpm, rad/s, rps). To being able to relate linear with rotational speeds.

Assessment: Correct calculations of circular speeds.

Law of inertia newton's first law: This law states that a body is rest or in constant speed will remain in that state unless an external force is exerted on it: gravity, wind or the movement of another body. Inertia is the resistance of any physical object to any change in its state of motion. This includes changes to the object's speed, direction, or state of rest.

Competencies: To determine what inertia is. Distinguish clearly between rest, constant speed and accelerated motion.

Facilities / Materials: gym or sports court, ball

\section{Exercises / Plays:}

1. Initially, one student is placed on the ground with a ball, in front of another student (figure 3). Without moving the ball, the teacher notices the fact that the ball remains at rest if not touched. I.e. the ball resists against any motion. Secondly, the teacher orders to kick the ball softly. Once, the ball is moving, the teacher notice 
the fact if no forces acts on the ball, the ball keeps is in constant motion.

Variants: kicking harder with larger separation between students, other objects, passes through the air.

Learning results: To understand the concept of inertia. Being able to determine the inertia of a body.

Assessment: Correct description of the inertia concept.

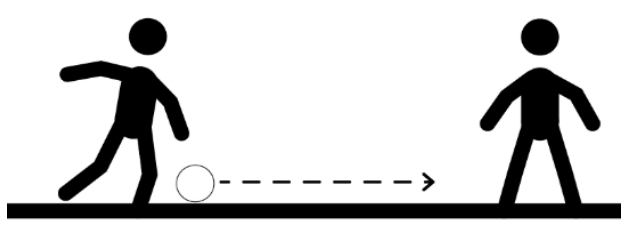

Figure 3. Students passing a ball.

Fundamental law of dynamics Newton's second law: When a force is applied to an object, it accelerates. This acceleration goes in the direction of force and it is proportional to its intensity and inversely proportional to the mass that moves. The mass can be seen as the resistance of a body to be accelerated

Competencies: Understand the relationship between mass, force and acceleration.

Facilities / Materials: Basketball, plastic ball, weighted ball.

\section{Exercises / Plays:}

1. Students are placed in pairs, one in front of the other. The space that separates them in meters is measured. One of them is asked to throw the ball to his partner without touching the ground (horizontal pass). The professor notice that the ball is accelerated from zero speed to constant speed by applying a force with the arms. Students may try to measure the final constant speed that the ball reaches after throwing. This speed gives feature of the acceleration provided. Variants: throw the ball faster or slower, through the ball with a bounce, use different balls with different weights.

Learning results: To relate the amount of force applied with the acceleration of the object.

Assessment: Correct analysis of the effect of the mass on the acceleration.

Law of action and reaction Newton's third law: The law of action and reaction can be easily understood because this effect is very common in our lives. When an object exerts a force on a second object, the second object exerts a force of equal magnitude and opposite direction on the first. We will use a funny game like the rope game to relate the commonly known phenomena with the physical law statement.

Competencies: Distinguish and identify clearly action and reaction. Understanding the unit of Newton.

Facilities / Materials: sports court, rope, skates

\section{Exercises / Plays:}

1. Initially, two students are placed on the ends of a rope (figure 4), at the same distance from the center of the string. Students are asked to start pulling at the same time. What do they experience?

Variants: competitions in teams.

2. Ground reaction force: Carry out previous process with skates and answer the same questions. What differences do you find? Which force is responsible of the reaction against the ground? The friction between the ground and the sports shoes is greater than the friction between the ground and the skates

Learning results: To identify correctly the forces that acts on a body, both reaction and action forces.

Assessment: Correct identification of both forces.

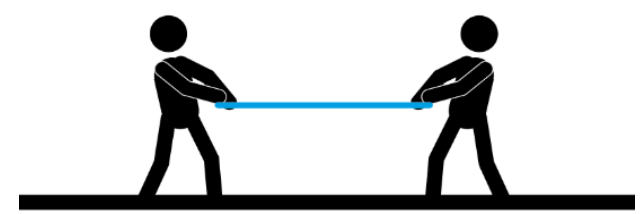

Figure 4. Students pulling a rope - action and reaction law.

Friction force and coefficient of friction: Friction force arises at anytime and anywhere in our lives. It is so common that students have difficulties to identify it as a force, likely gravitational one. The proposed activity will demonstrate the friction force relationship and also it will introduce the concept of friction coefficient.

Competences: Being able to identify friction as a force, and estimate the coefficient of friction between two surfaces. 
Facilities / Materials: sports court, rug or plastic, sand, balance

\section{Exercises / Plays:}

1. Place a rug or a plastic sheet on the ground. Then one student sits over the rug and a second student pulls from the rug (figure 5). They have to notice that friction is a force that opposes the movement. It is recommended to perform the exercise in a competitive way. Two pairs of students, for example, plays against other two. It is important to notice that the heavier is the student, the larger is the force. Even more, the teacher can measure the weight of different students on the rug, and try to relate the weight with the amount of force needed for the motion, qualitatively

Variants: several students on the rug, several students pull.

2. The coefficient of friction: It is proposed to repeat the exercise after spreading sand between ground and rug. Is this way the coefficient of friction will vary. Thus, the student will need more or less force for the same motion. The teacher must notice to the students this effect. The exact coefficient of friction can be estimated qualitatively. Variants: other lubricants can be added to the rug, other materials for the rug or plastic can be selected.

Learning results: To identify friction as a force, to calculate correctly the coefficient of friction

Assessment: Correct identification of friction force and coefficient of friction estimation.

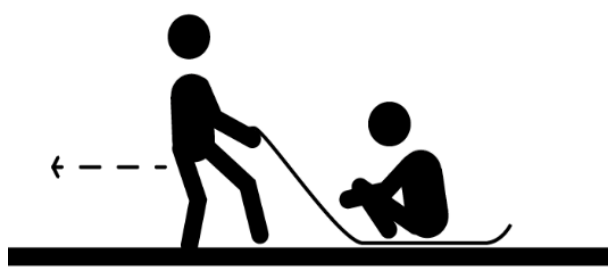

Figure 5. Student sitting on a rug and a classmate pulling.

Impulse: The impulse is the action of applying a certain force for a certain time. The final speed achieved by the body is proportional to its mass and the impulse applied to it. This phenomenon can be very well understood by means of a skateboarding and two students.

Competencies: Distinguish between force and impulse. Being able to estimate the value of an impulse.
Facilities / Materials: athletics track or sports court, chronometer, skateboard.

Exercises / Plays:

1. Measure and mark a distance around 3-5 meters. One student sits down on a skateboard several meters far from the initial mark and the other student places behind. A third student register times. At a certain moment, the second student starts to push the classmate on the skate. When the skateboard achieves the initial mark, the student releases the skateboard. The third student has to register the time during the pushing and the time that the skateboard needs to go through the marks. With this time, the students must calculate the speed after impulse, assuming that the speed of the skate is constant between marks, and the force of impulse, considering the mass of the student sit on the skate.

Variants: longer tracks, competitions in teams.

Learning results: To determine correctly the impulse force. To calculate final speeds.

Assessment: Correct calculations of the average impulse force.

Fluids: air - aerodynamic drag and Magnus force: Although it is well known that we breathe air, it is not so clear for the students that we live immersed in a fluid, like if we were always inside a swimming pool. As the viscosity of the air is very small, we hardly appreciate the drag during daily actions. The activities hereby proposed will dig into these concepts.

Competencies: Identify the aerodynamic drag force. Identify the Magnus force.

Facilities / Materials: sports court, towel or piece of cardboard, light plastic ball

\section{Exercises / Plays:}

1. Aerodynamic drag: One student holds a towel or a large piece of cardboard above the head. Then, he starts running. They have to notice that when running a force appear on the towel or cardboard opposing to their motion. If they run faster, the force will increase quadratically.

Variants: slower or faster running.

2. Magnus force: Initially each student is placed with a light ball (figure 6). They are asked to hit the ball and throw it forward. 
Where does the ball go? Perform the above process inducing in the ball a rotation. In the upper part of the sphere, where the relative velocity is lower, the surface of the sphere helps and accompanies the air particles, making the boundary layer remain attached to the ball for a longer time. In the lower part, on the other hand, the relative speed is greater and the boundary layer is released earlier. As a consequence, the outgoing airflow is diverted downwards. The ball will experience an upward force. In other words: if the air is deflected downwards, the ball will be deflected upwards.

Variants: Perform the previous process by shot a soccer ball on goal. Would this shot allow a goal from the corner (Olympic goal)?

Learning results: To identify correctly the aerodynamic drag force and the Magnus force.

Assessment: Correct identification of forces to air viscosity.

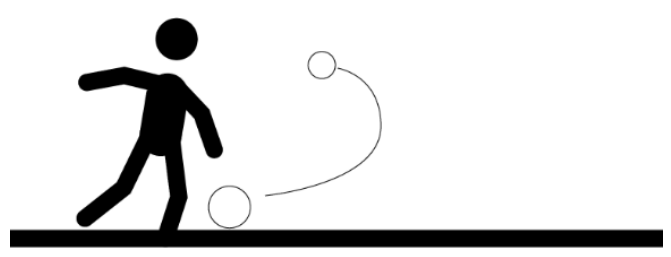

Figure 6. Student kicking a ball applying an effect in the ball.

\section{Normalizing mechanics technical language}

The second objective of this paper is to provide teachers resources for using mechanics technical language during their physical education lessons. The idea is to use technical language in the same manner, register or intonation that common language.

Students may seem annoyed, even disgusted at the start of the process. It will surely happen. However, as long as the teacher insists on the method, students will become more comfortable with the terms. It is important that first lessons using technical language are funny and relaxed in terms of physical education contents. Students maybe even laugh at the teacher for using this language. Never mind, with time and patience the technical language will deposit in their brains.

Sometimes, people that use technical language is seen as pretentious by those ignorant and ridiculous by those experts if it is not perfectly used. Not at all a problem, similar prejudgments happens by using a common language. The objective is not to become students into pedants or refined athletes, the objective is to trivialize technical language for a better assimilation. It is not needed even to be rigidly precise since it is intended to approach the terms to students. Using technical language even if the meaning is far from their curriculum objectives contributes to this familiarization. Associating the terms to some effect or phenomena is a highly recommended way of learning. Additionally, it is encouraging to teachers to make students explain actions, effects and events by using this new term. As long as the course goes forward, the students will deal with talking technically.

An extended list of general sports terms with their technical equivalent is provided next. Some terms have several definitions in a technical way. Specificities of certain sports are also given.

\section{General Actions}

Words that refer to actions commonly performed during sports are usually more concise than the technical description of the same. However, with the use of technical definitions, quick speech patterns can be achieved that are sufficiently agile to carry the class correctly. Below is the list of the most general actions and their technical language equivalents.

To Run: it is the most common action in sports. To run can be technically said as: to increase / gain horizontal velocity or kinetic energy; to get the benefit of friction at the shoes-floor interface to accelerate; to develop power; to convert chemical energy into kinetic energy; to displace into a fluid (air) with low viscosity.

To Jump: it refers to leap or spring off the ground or other support by a sudden muscular effort. To jump can be technically said as: increase / gain vertical velocity or potential energy; to convert muscles elastic energy into kinetic and then potential energy; to apply a force against the gravitational force; to avoid contact between shoes and floor. When the jump ends (fall) some other technical terms can be used: to recover kinetic energy from potential one (while falling); to feel the reaction force of ground against us; to absorb/dissipate kinetic energy; to damp motion; to apply a force/impulse to the ground.

To climb: it means to move on or proceed, using the hands and feet, on or from an elevated area. To climb can be physically said as: to gain/increase potential energy; to apply an impulse through hands of feet to the rest of the body; to get the benefit of friction to displace vertically; to move against gravity; to reduce the gravitational force differentially; to move to a lower atmospheric pressure environment.

To jog: to run at a slow, steady pace. Same terms than for to run applies. As jogging is easier to keep on time, we can use: to keep constant velocity; to overcome constant aero-dynamical drag force. 
To hit: it refers to the action of hit with the hands, kick if it is the case of the foot or with external element like rackets or bats, another object. To hit can technically describe as: apply a force to an object; provide impulse during with very short time; to accelerate an object by exerting a force; to transfer kinetic energy from the hitting element to the object. Depending on the type of hitting and the hitting object other alternatives can be used: provide motion or velocity; to pass from rest to constant velocity.

To swim: it is also one common practice during the high-school period. It can be described as: move through a viscous fluid; to displace volume and thus to float; displace on the water due to reaction forces; to overcome drag hydrostatic forces; to impulse water to obtain body acceleration.

To dive: in technical language to dive into the swimming pool can be said as to penetrate into a viscous fluid; to pass from a fluid (air) to another fluid (water); to displace a volume of water equal to the volume of our body; to pass to a larger pressure environment.

To stretch: it is referred to the action of stretching the muscles. More scientifically, stretching the muscles is to elongate the muscles slightly above the yield stress, this reduces the residual stresses on the muscle. Therefore, it can be said as: extent muscles above elastic limits; elongate muscles; apply a traction pressure; deform plastically.

To mobilize articulations: this process is recommendable before practicing any sports. More technically, to mobilize or to warm-up articulation can be said as: increase temperature; increase the lubrication to prevent joint wear; recover elasticity on the articulations.

To whistle: it is a common action when giving instructions during sports. For the point of view of a physicist it can be seen as: to vibrate the air and generate a high sound; to force a fluid going through a thin groove; to generate pressure sinusoidal waves. To throw: in this action, the thrown object typically moves in the air. Thus, it can be seen as a parabolic movement. Equivalents can be: induce the sphere to describe a parabolic motion; apply a long impulse and increase the kinetic energy of the solid; displace the solid through the fluid (air).

To shoot: this action is similar to throw but with higher velocities. Then, the same wording that for throw applies. As shoot is typically used when scoring we can use: apply an impulse to the object in such a way that the goalkeeper can not intersect it.

To score: in most of the sports is to pass a ball through the goal. More technically we can say: to trespass the sphere through the square-shaped structure or ringshaped structure.

To dribble: it means to avoid an opponent by changing the direction. Technical language for dribbling can be: to change abruptly the direction; to get the benefit of the inertia that your opponent has to change the direction before your opponent realizes, since the opponent needs time to decelerate or brake; the student can have enough time to overpass the opponent. Also, it can be seen as get benefit of friction for a sudden change of direction.

To catch: this intends to the action of receiving an object to continue properly the game. Technical equivalents are: absorb completely the kinetic energy of the element or to make the element decelerate until zero.

To pass: action of giving the ball to other person. Physically this means to apply force to the sphere or element to gain velocity towards the direction of the playmate position. If the pass is through the air: apply parabolic motion to reach the playmate position. Provide impulse to the object.

To serve: technically to serve can be said: to initiate the motion of the element/object by applying force. Impulse. To give kinetic energy to the object.

To bounce: this action takes place in almost all the sports involving balls. To bounce technically is to give motion to the sphere towards the ground and getting benefit of the elasticity of the ball, recover the potential energy. Another term can be to pass from potential, to kinetic and to elastic energy. When the ball does not bounce totally, we can state that the ground has absorbed part of the energy or that the ground has damped the element.

To push: this verb is very used in any lesson. It is proposed to replace to push by applying/exert force; compress; apply compressive load/force. Inversely, to pull can be replaced with applying/exert force towards oneself, traction; apply traction load/force.

To lift: this is almost a technical term for increase potential energy of a solid.

To drink: physically the action is to flow water into our body volume.

To balance: this applies to the action when an athlete keeps a static position of the body or some external element.

\section{General elements}

It is also convenient to use technical synonyms instead of the sporty name of the different elements used during Physical Education lessons.

- Ball - solid, object, punctual mass, sphere, pressurized volume,

- Bat - solid, cylinder, wood cylinder, object, rigid solid.

- Darts - arrow, projectile

- Dice - cube, solid, object

- Baseball - solid, object, punctual mass, wood sphere, pressurized volume, rigid solid 
- Basketball - elastic solid, solid, object, punctual mass, sphere, pressurized volume,

- Football - elastic solid, solid, object, punctual mass, sphere, pressurized volume,

- Fishing pole - long cylinder, pendulum

- Goal - square, rectangle, inside the perimeter.

- Basket / hoop - ring, circumference, inside the perimeter.

- Racket - solid, cylinder, wood cylinder, object, elastic solid.

\section{Specific Sports Vocabulary}

There are some specific actions and nouns for different sports that are worth to use into technical language. As the words are rarer, we have also included the definition.

\section{Athletics Vocabulary}

Baton: it is a short stick or tube passed from runner to runner in a relay race. We can name the baton as a cylinder, or relay mass.

Discus: a heavy, thick-centered disk; the sport of throwing the discus. This can also be named as a disk, plate or object in rotation. Object with one large moment of inertia around the axis and two other moments of inertia smaller.

Hammer: it is a 16-pound metal ball attached to a wire for throwing in an athletic contest; the sport of throwing the hammer. Moving mass, punctual moving mass or mass under parabolic motion can be used.

High jump: sport in which competitors jump over a bar that is raised until only one competitor can jump over it. A high jump can be described as a conversion from elastic energy of the muscles into potential. However, the kinetic energy of the jumper remains constant.

Javelin: a lightweight, spear-like object; the sport of throwing the javelin. It can be defined long bar mass, Long jump: A long jump can be described as a conversion from the elastic energy of the muscles into potential. As the same as high jump, the kinetic energy of the jumper remains constant.

Sprint: a short, fast race run over a distance of 400 meters or less. Terms like high power or supply the maximum of kinetic energy in a very short time can be used.

\section{Gymnastics Vocabulary}

Apparatus: equipment, such as horizontal bars, trampolines or the balls and ribbons used in rhythmic gymnastics. Each apparatus can be seen as a rigid structure and/or elastic structures.
Balance: the ability to keep your body steady and upright. Technical word is equilibrium or compensation.

Dismount: the act of jumping off an apparatus like the uneven bars at the end of a routine. Physically this means to lose the potential energy achieved during the jump.

Handstand: a position with your hands on the ground while your body and legs are upright in the air. It is possible to use the terms inverse the body orientation.

Landing: the last part of a dismount or jump, when the feet touch the ground. It is an impact against the ground and a damping of the kinetic energy of the vertical motion.

Pommel horse: an apparatus that has a thick beam with two handles on top that are used while performing. This apparatus is a rigid structure that reacts against the athlete weight.

Trampoline: a frame with material stretched across that you can bounce on. In terms of mechanics, a trampoline is a pendulum or an elastic surface.

Twist: a movement in which part of the body, or the whole body, turns around. Mathematically, a twist is a full rotation, full revolution or 360 degrees of rotation.

\section{Swimming Vocabulary}

Backstroke: a swimming stroke you can do on your back. To displace fluid (water) in the opposite direction:

Breaststroke: an easy stroke in which you move your legs like a frog. To develop the motion from impulse against the water.

Butterfly: a difficult stroke mostly used by competitive swimmers. This style requires a large amount of power with a very high force of the arms and low velocity.

Dolphin kick: a rhythmic leg movement only used in the butterfly stroke

Flutter kick: the leg movement used in freestyle and backstroke. Alternative motion of the legs propelling the fluid and obtaining thrust from the reaction force.

\section{Conclusion}

In this work, an innovative experimental and demonstrative pedagogical methodology is described. The methodology is based on two main pillars: embracing concepts while playing and normalizing technical language during pleasant moments.

In the first part, we present a complete didactic unit. We describe in detail exercises to be carried out by the physical education teacher, the mechanics teacher or jointly. These are simple exercises, with games and in which we relate the actions with the explanation of the physical phenomena that take place. 
In the second part, we present a list of technical vocabulary equivalent to slang words or sports language. The purpose of this compendium is that the teachers and therefore the students change their sports and colloquial language by the precise technical language. If this learning is done during a period of play or sports practice, in the medium term it will be more familiar to the student and thus less boring.

\section{References}

[1] I. Abrahams, M. Reiss, and R. Sharpe, "The assessment of practical work in school science.," Stud. Sci. Educ., vol. 49, no. 2, pp. 209-251, 2013.

[2] J. a. Zahorik, "Elementary and Secondary Teachers' Reports of How They Make Learning Interesting," Elem. Sch. J., vol. 96, no. 5, p. 551, 1996.

[3] T. Lyons and F. Quinn, Choosing Science: Understanding the declines in senior high school science enrolments. 2010.

[4] B. M. Jack and H. shyang Lin, "Making learning interesting and its application to the science classroom," Stud. Sci. Educ., vol. 53, no. 2, pp. 137-164, 2017.

[5] A. Fitzgerald, Science in primary schools: Examining the practices of effective teachers, vol. 9789460918. 2012.

[6] H. shyang Lin, F. Lawrenz, S. F. Lin, and Z. R. Hong, "Relationships among affective factors and preferred engagement in science-related activities," Public Underst. Sci., vol. 22, no. 8, pp. 941-954, 2013.

[7] B. M. Jack, L. Lee, K.-K. Yang, and H. Lin, "A Science for Citizenship Model: Assessing the Effects of Benefits, Risks, and Trust for Predicting Students' Interest in and Understanding of Science-Related Content," Res. Sci. Educ., vol. 47, no. 5, pp. 965-988, Oct. 2017.

[8] K. Larkin and R. Jorgensen, “I Hate Maths: Why Do We Need to Do Maths?' Using iPad Video Diaries to Investigate Attitudes and Emotions Towards Mathematics in Year 3 and Year 6 Students," Int. J. Sci. Math. Educ., vol. 14, no. 5, pp. 925-944, Jun. 2016.

[9] P. Potvin and A. Hasni, "Interest, motivation and attitude towards science and technology at K-12 levels: a systematic review of 12 years of educational research," Stud. Sci. Educ., vol. 50, no. 1, pp. 85-129, 2014.

[10] G. Pinto, "The Bologna Process and Its Impact on University-Level Chemical Education in Europe," J. Chem. Educ., vol. 87, no. 11, pp. 1176-1182, Nov. 2010.

[11] "Ley Orgánica 8/2013, de 9 de diciembre, para la mejora de la calidad educativa.".

[12] C. Jourand, D. Adé, C. Sève, J. Komar, and R. Thouvarecq, "Dynamics of student interactions: an empirical study of orienteering lessons in physical education," Phys. Educ. Sport Pedagog., vol. 23, no. 2, pp. 134-149, Mar. 2018.

\section{Acknowledgements}

We would like to thank Prof. María Teresa Jiménez Guzmán, Head of C.P. Clara Campoamor, Parla, Spain for being a reference and model of good teaching practices. 